G340(P) HEALTHCARE PROFESSIONALS' PERCEPTIONS AND EXPERIENCES REGARDING WHAT WORKS WELL IN THE DISCHARGE OF CHILDREN WITH MEDICAL COMPLEXITY: A QUALITATIVE SERVICE EVALUATION

S Ramshaw. Institute of Applied Health Research, University of Birmingham, Birmingham, UK

10.1136/archdischild-2020-rcpch.293

Aim This service evaluation explored healthcare professionals' perceptions of what works well in the discharge of children with medical complexity (CMC), within a tertiary paediatric centre. This sub-group of paediatric patients experience high healthcare resource usage, due to complex medical and social situations. Discharge is a critical opportunity to improve care quality, and $\mathrm{CMC}$ are at higher risk of poor discharge outcomes such as delays and hospital readmissions.

Methods This evaluation was informed by the 'Learning from Excellence' approach to quality improvement, by asking what works well to allow for both positive and negative opinions to be explored. 15 one-to-one interviews were conducted with a variety of individuals from the multi-disciplinary team directly involved in discharge planning for CMC. A topic guide aided the interviews, and thematic analysis of interview transcripts identified key themes.

Results 5 final themes were identified regarding areas that work well, alongside areas which may need improvement; Patient-centred Plan, Leadership for CMC, Supporting Families, Team Co-ordination, External Relationships. Tailoring discharge plans to individual patients and having a driver for coordinating discharge is perceived to facilitate a successful discharge. Families of CMC require constant support throughout discharge, achieved best by effective communication. Additionally, the capacity of families to care for their child has a perceived impact on the success of some discharges. A lack of team co-ordination between staff is a perceived barrier to discharge. Collaborative links between external services involved in discharge and the hospital helps the trust combat some barriers to discharge, with the need for links to other services to be built, specifically social care. Alongside theses five themes, three additional concurrent themes were elicited from the data; Communication, Capacity, and Collaboration.

Conclusion As the population of CMC is increasing, their pressure on healthcare resources grows. Their medical fragility makes transitioning home unpredictable, with a pressing need to optimise discharge. Numerous factors are perceived to work well when discharging $\mathrm{CMC}$, including supporting families and encouraging collaborative team working. The need to incorporate family capacity into planning and improve accountability of staff to discharge tasks can help accelerate this.

\section{G341(P) 10-YEAR ALLOGRAFT HISTOLOGY AND BIOPSYCHOSOCIAL HEALTH 20 YEARS AFTER PAEDIATRIC LIVER TRANSPLANTATION}

S Vimalesvaran, L Nevus, M Deheragoda, M Samyn, HV Melendez, N Heaton, A Dhawan. Paediatric Liver, GI and Nutrition Center, King's College Hospital, London, UK

\subsection{6/archdischild-2020-rcpch.294}

Aims Survival 20-years after paediatric liver transplantation is $>80 \%$. A correlation of this success is that long-term complications are becoming more evident. The aim of this study was to evaluate the long-term medical and psychosocial outcomes of paediatric post-liver transplant patients.

Methods Cohort study of paediatric liver transplant patients in a tertiary UK transplant centre, between 1985 and 2004. Patients surviving to 10 -years post-transplantation with normal liver biochemistry were included. Demographic data including indications for transplantation and complication rates were collected. All children underwent protocol liver biopsy at 10years. Electronic case notes were reviewed for long-term physical and psychosocial outcomes.

Results 84 patients survived to 10 years post-transplant. Median age at transplantation was 1.3 years, with median duration of follow-up of 20.2 years. Most common indication for liver transplantation was extrahepatic biliary atresia (51.2\%). Mortality rate after 10 years was $2 \%$. The observed acute and chronic rejection rates were $33.3 \%$ and $21.4 \%$, respectively.

A significant proportion (66.7\%) of children demonstrated mild graft fibrosis at 10-years, despite having normal liver biochemistry.

Long-term psychosocial consequences were significant. Roughly $26 \%$ of children had emotional and behavioral problems - depression and anxiety being the commonest. Higher rates of psychosocial disorders $(35.7 \%, \mathrm{p}=0.59)$ were seen in patients who required re-transplantation. Substance abuse including smoking and heavy alcohol consumption was lower than the national average $-9.5 \%$ and $3.6 \%$ respectively. Education and employment rates were comparable to the national average - 38\% and 27\%, respectively. In our cohort, 31\% were in transition clinics.

We report 23\% with chronic renal dysfunction 20-years post-transplantation, on standard immunosuppression regimens. eGFR values were calculated using cystatin-C values. Only 3 patients had a BMI of $>30$, with a mean BMI of 20.4 in our cohort.

Conclusion Whilst survival is excellent at 20 years, there is an emergent long-term impact of transplantation. Liver histology was abnormal in a significant proportion of our cohort despite normal liver biochemistry. Our findings demonstrate the importance of assessing physical health, specifically with regard to renal function and growth, as these children transition to adult services. The overarching impact of transplantation on the psychosocial wellbeing of survivors should form a key part of assessment post-transplantation.

\section{G342(P) CARDIAC FUNCTIONS IN CHILDREN WITH CELIAC DISEASE}

${ }^{1} \mathrm{~B}$ Haroun, ${ }^{1} \mathrm{AE}$ Eskander, ${ }^{2} \mathrm{R}$ Sobhy, ${ }^{3} \mathrm{Y}$ Farag, ${ }^{2} \mathrm{AF}$ AbdelMassih. ${ }^{1}$ Department of Pediatric Gastroenterology, Cairo University, Cairo, Egypt; ${ }^{2}$ Department of Pediatric Cardiology, Cairo University, Cairo, Egypt; ${ }^{3}$ Department of Pediatrics, Cairo University, Cairo, Egypt

\subsection{6/archdischild-2020-rcpch.295}

Aims Celiac disease (CD) associated cardiologic disorders is a growing concern. However, data regarding myocardial involvement in children with known CD are few. This study aimed at evaluating the subclinical impact of $\mathrm{CD}$ on the global myocardial function in Egyptian children with CD using Tissue Doppler imaging (TDI) and speckled tracking echocardiography (STE).

Patients and Methods Conventional two dimensional echocardiography was used to assess the myocardial function among 32 children with $\mathrm{CD}$ as well as 30 age and sex matched 
healthy controls. Advanced echocardiographic techniques were used to measure GLS, LV E/e' septal and LV E/e' lateral and RV E'/A' in both patients and controls and compare with the parameters measured by conventional echo. Additionally, the CD patients were classified in to 2 groups based on their diet and their cardiac functions were compared accordingly.

Results LVE/e' was significantly higher in CD than controls, $\mathrm{p}$ value $<0.0001$, suggesting diastolic dysfunction in cases compared to controls (table 1). In contrast, LV GLS (\%) was significantly lower in cases compared to controls with pvalue $<0.0001$, suggestive of subtle LV systolic dysfunction in cases with celiac disease. Moreover, both RV GLS (\%) and RV E'/A' were statistically lower CD patients than controls) denoting impaired both RV systolic and RV diastolic functions respectively. Finally, the LVEDV (ml) was lower in patients who were on gluten free diet for $\geq 6$ months Suggestive of positive reverse remodeling of the $\mathrm{LV}$ in celiac disease after implementation of gluten free diet.

\begin{tabular}{llll}
\multicolumn{4}{l}{ Abstract } \\
\hline Variable & $\begin{array}{l}\text { gluten-free diet for }<6 \\
\text { months }(n=14) \text { mean } \pm \text { SD }\end{array}$ & $\begin{array}{l}\text { gluten-free diet for } \geq 6 \\
\text { months }(n=18) \text { mean } \pm \text { SD }\end{array}$ & P-value* $^{*}$ \\
\hline LVEDV (ml) & $78.89 \pm 4.07$ & $72.71 \pm 4.86$ & 0.001 \\
\hline
\end{tabular}

Conclusions Subclinical myocardial dysfunction of both ventricles occurs in children with CD. This observed dysfunction seems to follow the same pattern of improvement of the gut pathology when gluten free diet is implemented.

\section{G344(P) VANCOMYCIN-INDUCED ACUTE KIDNEY INJURY IN NON-CRITICALLY ILL CHILDREN AT A TERTIARY PAEDIATRIC HOSPITAL}

${ }^{1} \mathrm{DE}$ Redding, ${ }^{2,3} \mathrm{~S} J$ McWilliam. 'Medical School, University of Liverpool, Liverpool, UK; ${ }^{2}$ Department of Women's and Children's Health, University of Liverpool, Liverpool, UK; ${ }^{3}$ MRC Centre for Drug Safety Science, University of Liverpool, Liverpool, UK

\subsection{6/archdischild-2020-rcpch.296}

Aims Vancomycin is known to have potential to cause nephrotoxicity. This retrospective audit aimed to describe the epidemiology of acute kidney injury (AKI) in non-critically ill paediatric patients exposed to treatment with intravenous vancomycin, at a tertiary paediatric hospital in the UK.

Methods The electronic medical records of all inpatients at a tertiary paediatric hospital who had one or more vancomycin drug levels measured between 1st October 2018 and 31st March 2019 were reviewed. All patients who received a course of intravenous vancomycin outside of the paediatric intensive care unit were included. Vancomycin-induced AKI was defined as any stage of AKI by the KDIGO criteria (an increase in serum creatinine of more than 50\% from baseline) occurring during, or in the 7 days following, vancomycin exposure.

Results Over six months, 74 courses of intravenous vancomycin were administered to a total of 60 children. There were 12 episodes of vancomycin-induced AKI in 12 children (7 $(58.3 \%)$ stage $1,3(25 \%)$ stage $2,2(16.7 \%)$ stage 3$)$. The incidence of vancomycin-induced AKI was 16.2 per 100 courses and 20 per 100 children.

\section{Abstract G344(P) Table 1}

\begin{tabular}{lll}
\hline & No AKI (n=62) & AKI ( $\mathbf{n = 1 2 )}$ \\
\hline Age (mean, SD) & $2.65(4.99)$ & $7.66(5.75)$ \\
Male (N,\%) & $39(62.9 \%)$ & $7(58.3 \%)$ \\
Concomitant nephrotoxins (mean, SD) & $0.71(0.86)$ & $1.58(1.24)$ \\
Trough >20 (N,\%) & $8(12.9 \%)$ & $2(16.7 \%)$ \\
Days of Vancomycin (Mean, SD) & $4.13(2.24)$ & $4.5(4.17)$ \\
Initial Vancomycin dose (mg/kg/day) (mean, SD) & $24.33(12.86)$ & $25.85(7.62)$ \\
Peak Vancomycin dose (mg/kg/day) (mean, SD) & $37.55(17.89)$ & $41.71(17.72)$ \\
Previous renal disease (N,\%) & $12(19.4)$ & $2(16.7 \%)$ \\
Previous AKI (N,\%) & $6(9.7 \%)$ & $5(41.7 \%)$ \\
Previous cardiac bypass (N,\%) & $16(25.8 \%)$ & $4(33.3 \%)$ \\
\hline
\end{tabular}

Concomitant nephrotoxin exposure was greater in the AKI group (table 1). The most frequent concomitant nephrotoxins associated with vancomycin-induced AKI were diuretics (furosemide and spironolactone) $(n=6,50 \%)$, piperacillin-tazobac$\operatorname{tam}(n=3,25 \%)$ and teicoplanin $(n=3,25 \%)$.

Conclusion Incidence of vancomycin-induced acute kidney injury in children is high, as seen in other studies. Patients on vancomycin should receive close monitoring for the development of AKI, including daily measurement of serum creatinine. Modifiable risk factors, such as exposure to concomitant nephrotoxins, should be minimised.

\section{G345(P) ABSTRACT WITHDRAWN}

\section{G346(P) ABSTRACT WITHDRAWN}

\section{G348(P) URSODEOXYCHOLIC ACID IMPROVES METABOLIC OUTCOMES IN THE FETUS, PLACENTA AND OFFSPRING OF HYPERCHOLANEMIC PREGNANCY}

${ }^{1} \mathrm{~A}$ Qadri, ${ }^{2} \mathrm{G}$ Papacleovoulou, ${ }^{2} \mathrm{LB}$ Manna, ${ }^{2} \mathrm{~V}$ Formigo-Pataia, ${ }^{2} \mathrm{~S}$ Abu-Hayyeh, ${ }^{2} \mathrm{P}$ Dixon, ${ }^{2}$ F Flaviani, ${ }^{2} \mathrm{C}$ Williamson. ${ }^{1}$ Institute of Reproductive and Developmental Biology, Imperial College London, London, UK; ${ }^{2}$ Maternal and Fetal Disease Group, Division of Women's Health, Kings College London, London, UK

\subsection{6/archdischild-2020-rcpch.297}

Aims Perturbations in the intrauterine environment can result in lifelong consequences for fetal metabolic health. We previously showed that in humans, intrahepatic cholestasis of pregnancy (ICP) can predispose offspring to metabolic disease, likely due to a combination of the effects of increased bile acids (BA), maternal dyslipidemia and deranged maternal and fetal lipid homeostasis. Ursodeoxycholic acid (UDCA) is a well-established treatment for maternal ICP. We aimed to address whether UDCA can also prevent the adverse metabolic effects of ICP in the offspring and fetoplacental unit.

Methods Women with ICP \pm UDCA treatment and controls with uncomplicated pregnancy were recruited for epigenome wide association studies which were performed on maternal and venous umbilical cord blood. Animal studies 